ISSN electrónico: 2445-1355

DOI: http://dx.doi.org/10.14201/fj2019423140

\title{
EVALUACIÓN DE LA MEDICACIÓN DE RIESGO EN UNA RESIDENCIA DE ANCIANOS
}

\section{Evaluation of High-Alert Medications in Older Residents in a Long Term Care Facility}

Patricia MALDONADO FERNÁNDEZ DE GATTA; María del Mar FERNÁNDEZ DE GATTA GARCÍA

Farmacia Fernández de Gatta. C/ Libertad, 73, Béjar. Salamanca

Correo-e: farmaciafernandezdegatta@gmail.com

RESUMEN: La identificación de medicamentos de alto riesgo en pacientes crónicos (MARC) polimedicados como los ancianos constituye una estrategia útil para aumentar la seguridad de la farmacoterapia. Con ese fin en el trabajo se aplica el listado MARC en una población de 103 ancianos ingresados en una residencia vinculada a una farmacia comunitaria. La identificación se realiza en la dispensación mediante receta electrónica detectándose simultáneamente posibles interacciones del tratamiento de cada paciente. Posteriormente se revisan específicamente los medicamentos más habituales de prescripción inadecuada como son benzodiacepinas, estatinas o IBP.

En el estudio se constata que más del $90 \%$ de la población tiene prescritos más de un MARC así como que el número de MARC prescritos $\mathrm{y}$ de potenciales interacciones se incrementa cuando el paciente pertenece al subgrupo de gran polimedicado (> 10 medicamentos). Sin embargo esas variables no varían en función de la edad o sexo del paciente. Las categorías de medicamentos MARC más prevalentes correspondieron a antiagregantes, benzodiacepinas, AINES y diuréticos. Estos dos últimos grupos justifican gran parte de las interacciones detectadas.

La estrategia utilizada en el estudio puede ser útil para introducir los servicios profesionales farmacéuticos en este tipo de entorno clínico.

Ediciones Universidad de Salamanca / @@ఠ $\quad$ FarmaJournal, vol. 4, núm. 2 (2019), pp. 31-40 
Palabras clave: Medicación de alto riesgo; residencias de ancianos; interacciones; servicios profesionales farmacéuticos.

SUMMARY: The identification of high-risk medications in polymedicated chronic patients as the elderly is a useful strategy to increase the safety of pharmacotherapy. To this end, the MARC list is applied in a population of 103 elderly people in a long term care facility linked to a community pharmacy. The identification is made at the dispensation act by electronic prescription, detecting simultaneously possible interactions of the patient's treatment. Moreover, the most usual medications with inadequate prescriptions, such as benzodiazepines, statins or PPIs, are specifically reviewed.

The study found that more than $90 \%$ of the population has more than one MARC prescribed and that the number of MARC prescribed and potential interactions increases when the patient is categorized as severe polymedicated ( $>10$ drugs). However, these variables do not vary according to the age or sex of the patient. The most prevalent MARC medications were Antiaggregants, benzodiazepines, NSAIDs and diuretics. These last two groups justify a large part of the interactions detected.

The strategy used in the study can be useful to introduce professional pharmaceutical services in this type of clinical environment

Key words: High risk medication; nursing homes; interactions; pharmaceutical professional services.

\section{INTRODUCCIÓN}

La prescripción farmacológica es la intervención médica más utilizada para mejorar la salud y evitar que sea inadecuada debe ser una prioridad compartida por todos los profesionales sanitarios.

Un medicamento es prescrito adecuadamente cuando hay evidencias claras de su uso en una indicación determinada, presenta buena tolerancia en la mayoría de pacientes y, su relación coste/efectividad es favorable (Galván-Banqueri et al., 2014). Además, la prescripción adecuada en ancianos debe considerar la esperanza de vida y evitar terapias preventivas con pronóstico de corta supervivencia (DelgadoSilveira et al., 2009). Una prescripción es potencialmente inapropiada (PPI) si el riesgo de efectos adversos es superior al beneficio clínico, existiendo alternativas terapéuticas más seguras y/o eficaces (Tommelein et al., 2015). El concepto incluye:

- Uso de medicamentos con mayor frecuencia o duración de la indicada.

- Elevado riesgo de interacciones. 
- Mala selección del medicamento o de su dosis.

- Duplicidades terapéuticas.

- Omisión en la prescripción de medicamentos indicados.

Un 50-60 \% de la población geriátrica residente en centros sociosanitarios recibe uno o más medicamentos inapropiados como parte de su medicación habitual (Storms et al., 2017). La PPI en ancianos es un problema de salud pública, relacionado con mayor morbimortalidad y aumento en el uso de recursos sanitarios. Existen métodos para su detección clasificados en implícitos (basados en el juicio clínico como el Medication Appropriateness Index) y explícitos (basados en criterios predefinidos como los STOPP-START). Esta metodología útil en diversos ámbitos sanitarios (hospitales, residencias, atención primaria, farmacias...) permite evaluar la calidad de la prescripción, pero es laboriosa, requiere tiempo y suficiente información, usándose más para evaluación o investigación que en la práctica clínica diaria (Mud Castelló F et al., 2014; Nicieza-García et al., 2016).

Los pacientes con patologías crónicas y polimedicados, particularmente los ancianos, son especialmente vulnerables a errores de medicación y por ello objetivo prioritario de estrategias de mejora de seguridad. Entre ellas se incluye detectar medicamentos de alto riesgo (MAR) que causan la mayoría de errores de medicación con consecuencias graves para los pacientes. El concepto surgió en el ámbito hospitalario, pero la lista MARC (Medicamentos de alto riesgo en pacientes crónicos) buscó ser útil en atención primaria para priorizar la implantación de prácticas seguras con medicamentos en paciente crónicos (Ministerio de Sanidad, Servicios Sociales e Igualdad, 2014).

En este contexto, la farmacia comunitaria puede tener un papel clave en la atención farmacéutica y prestación de servicios en residencias. El documento sobre Buenas Prácticas en Farmacia Comunitaria relacionado con la Prestación de Servicios Profesionales Farmacéuticos (SPF) en centros sociosanitarios o residenciales establece entre sus funciones «Revisar la medicación del residente, identificar problemas relacionados con los medicamentos y contribuir a prevenir o evitar los resultados negativos asociados a su uso, en coordinación con los médicos y el resto de miembros del equipo asistencial del centro». El listado MARC apenas ha sido evaluado con ese fin y sin embargo puede suponer una aproximación inicial a la prestación de ese tipo de SPF (Domínguez Sánchez-Migallon et al., 2017; Casas-Pérez et al., 2018).

Los objetivos del trabajo son utilizar el listado MARC en una población de pacientes ancianos ingresados en una residencia de ancianos y evaluar los resultados obtenidos para establecer si esta estrategia es factible en este entorno clínico contribuyendo a aumentar la concienciación de los riesgos de la prescripción inadecuada y a mejorar la evaluación de la farmacoterapia. 


\section{MATERIALES y MÉTODOS}

Estudio observacional descriptivo transversal realizado en una farmacia comunitaria vinculada a una residencia de ancianos pública. La población diana incluyó pacientes mayores de 65 años atendidos en el mismo con al menos un tratamiento crónico (más de tres meses de duración) incorporado en receta electrónica. La recogida de información se realizó el primer trimestre del 2018.

Las variables estudiadas fueron:

- Independientes: edad, sexo y número total de medicamentos prescritos. Para el análisis de datos se crearon: dos categorías: gran polimedicado ( $\geq 10$ medicamentos) y edad ( $\geq 85$ años).

Figura 1. Listado MARC.

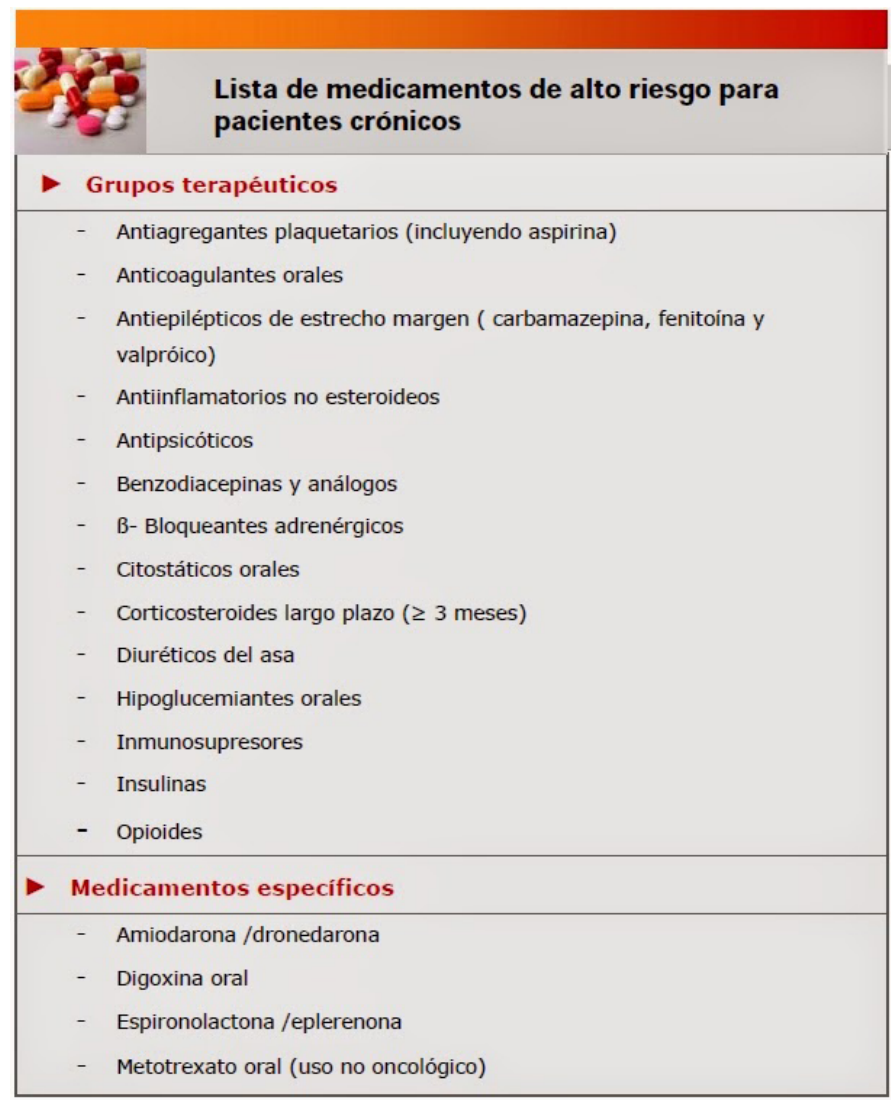


- Dependientes: número y tipo de MAR mediante aplicación del listado MARC (Figura 1) a cada paciente. Además se recogió la opción específica prescrita de IBP, estatinas, diuréticos y benzodiacepinas dada su frecuente asociación con problemas relacionados con la medicación y PPI (Revisión específica de la prescripción). Para el análisis de datos se evaluaron las variables: número y tipo de interacciones potenciales detectadas por paciente con BOT PLUS, número y tipo de duplicidades y recomendaciones terapéuticas para aumentar la seguridad de la terapia.

Los datos se recogieron de la hoja de medicación incluida en la receta electrónica del paciente en la primera dispensación solicitada. Se confeccionó una hoja de recogida de datos disociada por paciente (Microsoft Excel 2007) que posteriormente se migró al programa SPSS versión 17.0.

El análisis estadístico descriptivo se realiza mediante tablas de frecuencias o distribución porcentual para las variables cualitativas y con la media y desviación estándar en las cuantitativas. El análisis comparativo con la prueba chi-cuadrado para variables cualitativas y mediante t-Student o U-Mann Whitney para las cuantitativas (ANOVA o Kruskal Wallis si se comparan más de dos variables cuantitativas). Se consideró significativa una $\mathrm{p}<0,05$.

\section{Resultados}

Se evaluaron 114 pacientes (78 mujeres/36 hombres) de entre 65-101 años (media 88,9 \pm 6 años). Un $47 \%$ se incluyó en la categoría de polimedicación severa. La tabla 1 recoge las características generales de la prescripción crónica.

TABLA 1. Características generales de la prescripción crónica en la población.

\begin{tabular}{|l|c|c|}
\hline VARIABLE & Media \pm DE & Rango \\
\hline $\begin{array}{l}\text { N. }{ }^{\circ} \text { total de medicamentos pres- } \\
\text { critos por paciente }\end{array}$ & $8,9 \pm 3,5$ & $1-18$ \\
\hline $\begin{array}{l}\text { N. }{ }^{\circ} \text { de medicamentos MARC } \\
\text { prescritos por paciente }\end{array}$ & $2,6 \pm 1,6$ & $0-7$ \\
\hline $\begin{array}{l}\text { N. }{ }^{\circ} \text { de duplicidades detectadas por } \\
\text { paciente }\end{array}$ & $0,58 \pm 0,76$ & $0-3$ \\
\hline $\begin{array}{l}\text { N. }{ }^{\circ} \text { de interacciones potenciales } \\
\text { detectadas por paciente }\end{array}$ & $2,9 \pm 3,2$ & $0-13$ \\
\hline
\end{tabular}


La aplicación del listado MARC mostró que solo 12 pacientes (10,5\%) no tenían ningún medicamento prescrito incluido en dicho listado y en casi un $30 \%$ el número de medicamentos MARC prescritos fue de 4 o superior. Las categorías de medicamentos MARC más habituales en la población fueron antiagregantes plaquetarios $(31,5 \%)$, anticoagulantes orales (21,9\%), AINES (32,4\%), benzodiacepinas y análogos $(53,5 \%)$ y diuréticos del asa (42,1\%). Ningún paciente tenía prescritos medicamentos MARC específicos, excepto digoxina (7 pacientes). No se encontraron diferencias estadísticamente significativas para el número total de medicamentos prescritos y tipo MARC en función del sexo o edad y sí una estadísticamente significativa relación lineal entre el número de medicamentos

FIGURA 2. Influencia de la polimedicación sobre el número potencial de interacciones y sobre la prescripción de medicamentos de alto riesgo en pacientes crónicos.

(Diferencias estadísticamente significativas Kruskall-Vallis $\mathrm{p}<0.05$ ).

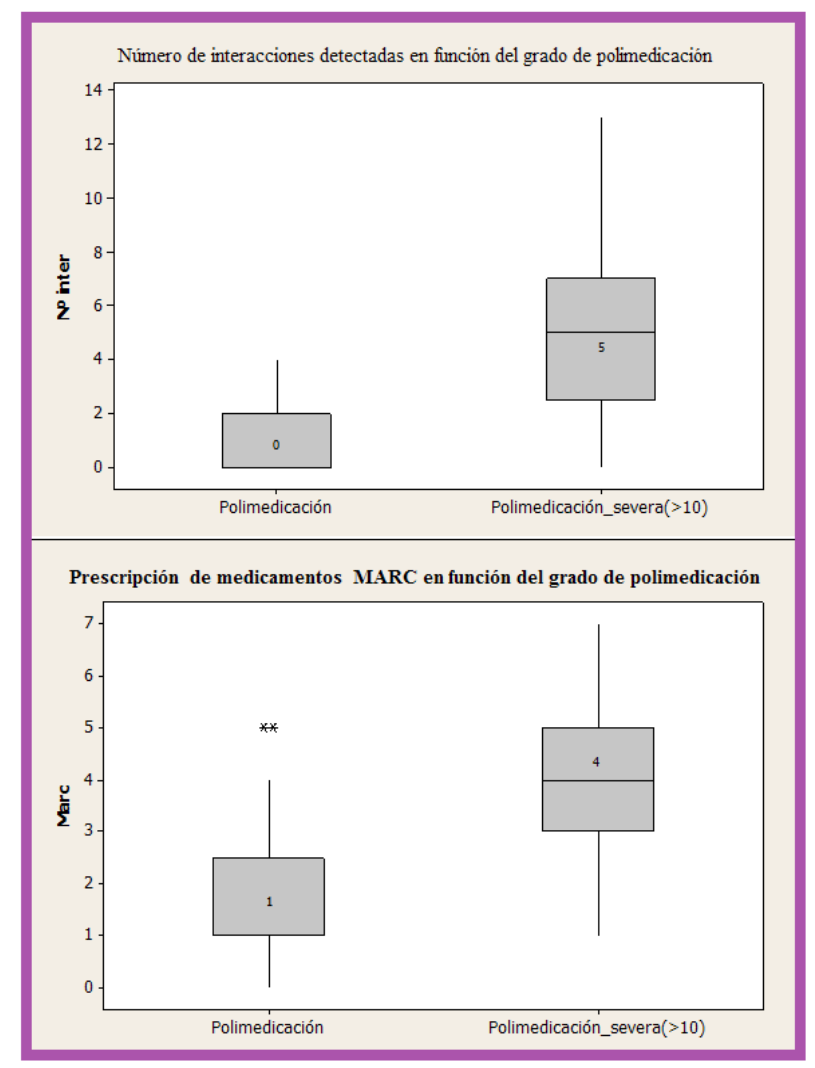

Ediciones Universidad de Salamanca / @@ FarmaJournal, vol. 4, núm. 2 (2019), pp. 31-40 
MARC detectados por paciente y el número total de medicamentos prescritos $\left(\mathrm{r}^{2}=0.52\right)$. Así la mediana de medicamentos MARC por paciente aumenta de $1 \mathrm{a}$ 4 en los pacientes con polimedicación severa (Figura 2).

El número de potenciales interacciones detectadas aumenta con el número de medicamentos prescritos y en pacientes con polimedicación severa el valor de la mediana es de 5 (Figura 2). Destaca la detección en dos pacientes de una interacción grave y bien documentada (triple whammy) debida a la combinación de IECA, diuréticos y AINES (Thomas MC, 2000) y dos interacciones no reconocidas por el programa BOT Plus (ciprofloxacino asociado a suplementos de calcio y omeprazol con hierro). Las interacciones más frecuentemente detectadas implicaron al uso de diuréticos junto a: IBP (47 pacientes), AINES (17 pacientes) e IECA (12 pacientes) y las relacionadas con la prolongación del intervalo QT (18 pacientes) o la combinación de AINES con IECA (15 pacientes).

Las duplicidades más habituales correspondieron a analgésicos (15 pacientes), benzodiacepinas (10 pacientes) y diuréticos (10 pacientes). La figura 3 muestra el análisis de la prescripción específica realizado respecto a IBP, estatinas, benzodiacepinas y diuréticos donde la categoría riesgo incluye: omeprazol; atorvastatina y simvastatina; diazepam, bromazepam, flurazepam; y diuréticos del asa respectivamente.

FIgURA 3. Resultados de la revisión específica de la prescripción.

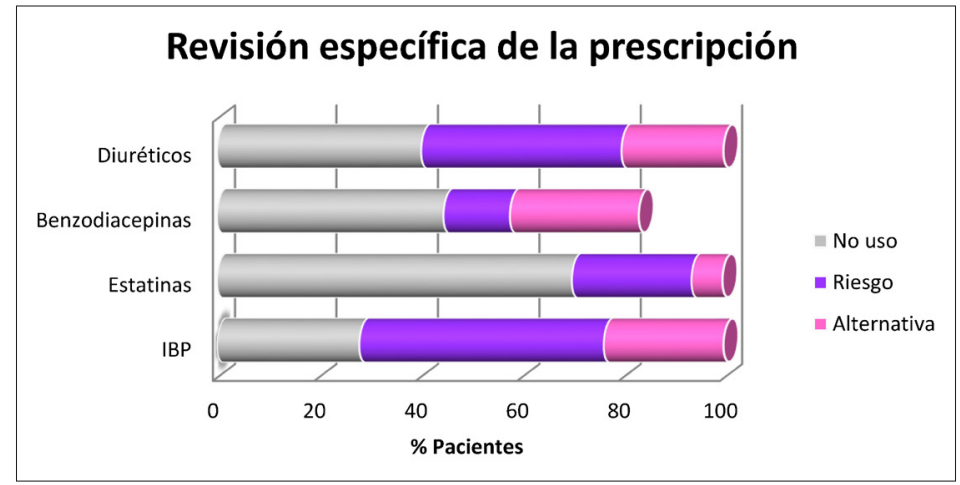

\section{Discusión}

Los farmacéuticos deben garantizar un uso seguro, responsable, efectivo y eficiente de los medicamentos, así como desarrollar servicios e intervenciones sanitarias que tengan como objetivo último optimizar los resultados en salud. 
En farmacias comunitarias las estrategias de promoción de salud y prevención de enfermedad deben centrarse en actividades con demostrada evidencia e incluidas en las prioridades de los programas sanitarios. En ese sentido, optimizar la farmacoterapia en pacientes con tratamientos crónicos, con especial atención a los ancianos polimedicados, parece una estrategia prioritaria y factible. La mayoría de estudios realizados en estas poblaciones para evitar potenciales problemas de seguridad relacionados con la prescripción, aplican otros criterios explícitos (Criterios Beers, STOPP/START) (Ubeda et al., 2012; Barris Blundell D., et al., 2015) y son pocos los que usan el listado MARC (Domínguez Sánchez-Migallon et al., 2017; Casas-Pérez et al., 2018).

La PPI es un problema de alta prevalencia en ancianos ingresados en residencias y guarda una estrecha relación con el número total de medicamentos prescritos (Storms et al., 2017; Ubeda et al., 2012). En este estudio el uso de MARC también aumenta con el número total de medicamentos prescritos y la prevalencia es casi del $90 \%$, dato probablemente relacionado con la herramienta utilizada. Destaca el uso de diuréticos, benzodiacepinas y AINES como medicación de alto riesgo más habitual, lo que coincide con datos de PPI en estudios similares.

El listado MARC ofrece ventajas frente a otros criterios explícitos: es más generalizable, al referirse a grupos farmacológicos y a pocos medicamentos específicos, no requiere conocer el diagnóstico o la historia clínica y el tiempo de aplicación es mínimo una vez familiarizado con la lista. Sin embargo, no considera potenciales interacciones, duplicidades terapéuticas u omisiones de tratamiento beneficiosas para el paciente. Estas deficiencias se compensaron en el estudio evaluando interacciones y duplicidades de modo separado. Respecto a las interacciones se estableció un mayor n. $^{\circ}$ medio de potenciales interacciones que otros estudios con similar grado de polimedicación probablemente debido a diferencias metodológicas (CheckTheMeds®. vs BOT Plus) (Marín-Gorricho et al., 2017). Es destacable, por el mayor riesgo de interacciones, el elevado uso de omeprazol, atorvastatina y simvastatina o diazepam frente a mejores alternativas de su misma clase terapéutica. Las recomendaciones potenciales para mejorar la adecuación de los tratamientos y evitar potenciales problemas de seguridad supusieron en la mayor parte de los casos controles clínicos (analíticas, ECG, monitorización...) y seguimiento u observación del paciente y solo minoritariamente la posible modificación del tratamiento.

El estudio tiene limitaciones, como desconocer la situación funcional y socio-familiar o las patologías de los pacientes. La información se obtuvo de la receta electrónica que permite identificar adecuadamente la prescripción pero, en ocasiones, se constató la no utilización de fármacos incluidos en la misma. En el estudio no se realizó ningún tipo de intervención, por lo que los posibles beneficios clínicos son solo teóricos. No obstante, se adecua al principal objetivo 
del proyecto MARC: «implantación de prácticas seguras para mejorar la utilización de medicamentos de alto riesgo y evitar daños innecesarios a los pacientes». Las prácticas contemplan medidas de actuación en todos los procesos de uso de medicamentos (alertas en sistemas de ayuda a la prescripción, protocolos de seguimiento específicos o de revisión periódica del tratamiento, elaboración y difusión de procedimientos de dispensación segura en oficinas de farmacia, incluyendo medidas o precauciones para evitar los errores detectados) y, por tanto, involucran a los distintos profesionales que intervienen. La lista MARC es útil, pero por sí sola no va a reducir los riesgos con estos medicamentos. Para ello es preciso actuar de modo que todos aquellos que trabajan con pacientes crónicos desarrollen e impulsen la implantación de buenas prácticas, proporcionando una asistencia cada vez más segura».

La estrategia utilizada en este estudio parece factible con esa finalidad, ya que a diferencia de otros criterios explícitos el listado MARC no requiere ningún recurso informático ni formación especializada y por su concreción puede usarse sin problemas para revisar la medicación en el acto de dispensación. La detección de posibles interacciones es inmediata con el programa utilizado en la propia dispensación. Esto puede permitir la posterior intervención del farmacéutico con el resto del equipo asistencial del centro para prevenir o evitar los resultados negativos asociados al uso de los medicamentos y conseguir un uso racional de los mismos.

\section{Conclusiones}

La alta prevalencia de medicación de alto riesgo y de posibles interacciones encontradas en este estudio sugieren que un programa de revisión y adecuación de la medicación en residencias de ancianos vinculadas a farmacias comunitarias podría aumentar la efectividad de la farmacoterapia en este sector de la población.

La introducción de una herramienta sencilla como el listado MARC en la evaluación y seguimiento de los tratamientos farmacológicos es factible de realizar en el entorno de la farmacia comunitaria y puede mejorar la calidad asistencial de los pacientes ingresados en residencias.

La estrategia utilizada en este estudio puede ser útil para potenciar el análisis y la revisión de los tratamientos y poder introducir este tipo de SPF en las farmacias vinculadas a residencias de ancianos.

\section{Bibliografía}

Barris Blundell D. Revisión de medicación según criterios STOPP/START en pacientes mayores del servicio de sistema personalizado de dosificación de medicamentos de una farmacia comunitaria. Farmacéuticos Comunitarios. 2015; 7(2):31-36. 
Casas Pérez P, Álvarez Schoendorff J, Herrerías Esteban G. Utilización del Listado MARC en la identificación de pacientes crónicos en tratamiento con medicamentos de alto riesgo en farmacia comunitaria. Farmacéuticos Comunitarios. 2018; 10 (Suplemento 1).

Delgado-Silveira E, Muñoz-García M, Montero-Errasquin B, Sánchez-Castellano C, Gallagher P, Cruz-Jentoft AJ. Prescripción inapropiada de medicamentos en los pacientes mayores: los criterios STOPP/START. Rev Esp Geriatr Gerontol. 2009; 44(5):273-9.

Documento de Buenas Prácticas en Farmacia Comunitaria en España. Disponible en: http://www.portalfarma.com/Profesionales/Buenas-practicas-profesionales/Paginas/ Buenas-practicas- Farmacia-Comunitaria.aspx

Domínguez Sánchez-Migallon P. Aplicación del listado Marc a pacientes polimedicados de un Centro de Salud. Rev Clin Med Fam. 2017; 10(1):5-11.

Galván Banqueri M, Alfaro Lara ER, Rincón Gómez M, Rivas Covas PC, Vega Coca MD, Nieto Martín MD. Factors related with the appropriateness of pharmacological treatment in polypathological patients. Farm Hosp. 2014; 38(5):405-10.

Marín-Gorricho R, Casajus-Navasal A, Carrillo-Acevedo L, Serrano-Pérez A, BarajasSantos MT, Hurtado-Gómez MF. Análisis de la prescripción potencialmente inadecuada según los criterios STOPP/START en un centro sociosanitario. Pharm Care Esp. 2017; 19(6):499-520.

Mud Castelló F, Mud Castelló S, Rodríguez Moncho MJ, Ivorra Insa MD, Ferrándiz Manglano ML. Detección de prescripciones potencialmente inapropiadas en pacientes ancianos: estudio descriptivo en dos farmacias comunitarias. Farmacéuticos Comunitarios. 2014; 6(2):20-26.

Nicieza-Garcia ML, Salgueiro-Vazquez ME, Jimeno-Demuth FJ, Manso G. Criterios de Beers versus STOPP en pacientes mayores, polimedicados y residentes en la comunidad. Farm Hosp 2016; 40(3):150-64.

Proyecto MARC. Elaboración de una lista de medicamentos de alto riesgo para los pacientes crónicos. Madrid.

Storms H, Marquet K, Aertgeerts B, Claes N. Prevalence of inappropriate medication use in residential long-term care facilities for the elderly: a systematic review. Eur J Gen Pract 2017; 23(1):69-77.

Thomas MC. Diuretics, ACE inhibitors ans NSAIDs-the triple whammy. Med J Austr. 2000; 172:184-5.

Tommelein E, Mehuys E, Petrovic M, Somers A, Colin P, Boussery K. Potentially inappropriate prescribing in community-dwelling older people across Europe: a systematic literature review. Eur J Clin Pharmacol. 2015; 71:1415-27.

Ubeda M, Ferrandiz ML, Maicas N, Gomez C, Bonet M, Peris JE. Potentially inappropriate prescribing in institutionalised older patients in Spain: the STOPP-START criteria compared with the Beers criteria. Pharmacy Practice. 2012; 10(2):83-91. 